\title{
Charge and Spin Transport in the One-dimensional Hubbard Model
}

\author{
Shi-Jian Gu ${ }^{1,2}$, N. M. R. Peres ${ }^{3}$, J. M. P. Carmelo ${ }^{4}$ \\ ${ }^{1}$ Department of Physics and Institute of Theoretical Physics, The Chinese University \\ of Hong Kong, Hong Kong, China \\ ${ }^{2}$ Zhejiang Institute of Modern Phys, Zhejiang University, Hangzhou, 310027, China \\ ${ }^{3}$ Center of Physics and Physics Department of the University of Minho, School of \\ Sciences, Campus of Gualtar, P-4710-057 Braga, Portugal \\ ${ }^{4}$ GCEP-Center of Physics, University of Minho, Campus Gualtar, P-4710-057 Braga, \\ Portugal \\ E-mail: sjgu@phy.cuhk.edu.hk
}

\begin{abstract}
In this paper we study the charge and spin currents transported by the elementary excitations of the one-dimensional Hubbard model. The corresponding current spectra are obtained by both analytic methods and numerical solution of the Bethe-ansatz equations. For the case of half-filling, we find that the spin-triplet excitations carry spin but no charge, while charge $\eta$-spin triplet excitations carry charge but no spin, and both spin-singlet and charge $\eta$-spin-singlet excitations carry neither spin nor charge currents.
\end{abstract}

PACS numbers: 05.60.Gg, 72.10.-d, 71.27.+a 


\section{Introduction}

Recently, there has been a renewed interest in the unusual transport and spectral properties of nanotubes, ballistic wires, and quasi-one-dimensional (1D) compounds $[1,2]$. Quantum effects are strongest at low dimensionality leading to unusual phenomena such as charge-spin separation at all energies [2] and persistent currents in mesoscopic rings [3]. Thus, the further understanding of the transport of charge in low-dimensional correlated systems and materials is a topic of high scientific interest.

There is numerical evidence of a fundamental difference between the transport properties of integrable and nonintegrable 1D interacting quantum systems: at finite temperatures, $T>0$, the integrable systems behave as ideal conductors in the metallic quantum phases and as ideal insulators in the insulating phases, with the concepts of an ideal insulator and conductor defined in Ref. [4]. In contrast, the nonintegrable 1D interacting systems are generic conductors and activated ones in the metallic and insulating phases, respectively. While in the trivial case of 1D integrable systems whose Hamiltonians commute with the current operator the ideal insulating and conducting behaviors are easy to confirm, there is the expectation that such ideal behaviors might also occur in 1D integrable quantum systems whose Hamiltonian does not commute with that operator, such as the 1D Hubbard model $[5,6,7]$. However, the studies of Ref. [8] rely on the generalization of the thermodynamic Bethe-ansatz equations introduced in Ref. [7] to the model in a presence of a vector potential [9] and seem to reveal that for half filling the 1D Hubbard model does not remain an ideal insulator for $T>0$, in contrast to the general predictions and expectations of Ref. [4].

Solvable lattice models such as the 1D Hubbard model $[5,6,7,10]$ and the XXZ chain [11] are often used as toy effective models for the study of the unusual properties of quasi-1D compounds [12, 13]. Although the 1D Hubbard model was diagonalized long ago $[6,7]$ by means of the coordinate Bethe ansatz (BA) $[14,15]$, the involved form of BA wave functions has prevented the full calculation of dynamic response functions. The study of the asymptotic of correlation functions and of the low-energy dynamical properties was performed by combining the BA solution with other methods, such as conformal-field theory [16], bosonization [17, 18], the pseudo-particle formalism[19], and scaling methods [20].

In this paper, we study the charge and spin currents carried by the elementary lowenergy and finite-energy excitations of the 1D Hubbard model. The paper is organized as follows: In Sec. II we summarize the basic information about the model and the BA solution needed for our study; The energy and current spectra of the elementary excitations is the subject of Sec. III. Finally, in Sec. IV we present the concluding remarks. 


\section{The model and its Bethe-ansatz solution}

The Hamiltonian of the 1D Hubbard model on a periodic $N_{a}$-site chain reads,

$$
\mathcal{H}=-t \sum_{j, \sigma}\left(c_{j, \sigma}^{\dagger} c_{j+1, \sigma}+c_{j+1, \sigma}^{\dagger} c_{j, \sigma}\right)+U \sum_{j}\left(n_{j, \uparrow}-1 / 2\right)\left(n_{j, \downarrow}-1 / 2\right),
$$

where the operator $c_{j, \sigma}^{\dagger}$ (and $c_{j, \sigma}$ ) creates (and annihilates) an electron of spin projection $\sigma$ at the site of index $j=1,2,3, \ldots, N_{a}$ and $n_{j, \sigma}=c_{j, \sigma}^{\dagger} c_{j, \sigma}$ is the number operator at the same site. We use units of lattice constant one such that $L=N_{a}$, where $L$ is the system length. We denote the electron number and the spin-projection $\sigma$ electron number by $N$ and $N_{\sigma}$, respectively, such that $N=\left[N_{\uparrow}+N_{\downarrow}\right]$. Moreover, we denote the states spin and $\eta$-spin values by $S$ and $\eta$, respectively.

The model as written in Eq. (1) has both a spin and a $\eta$-spin $S U(2)$ symmetry $[21,22,23,24]$. The generators of the $\eta$-spin symmetry are given by,

$$
\begin{aligned}
& \eta=\sum_{j=1}^{L}(-1)^{j} c_{j \uparrow} c_{j \downarrow}, \quad \eta^{\dagger}=\sum_{j=1}^{L}(-1)^{j} c_{j \downarrow}^{\dagger} c_{j \uparrow}^{\dagger}, \\
& \eta^{z}=\frac{1}{2} \sum_{j=1}^{L}\left(n_{j \downarrow}+n_{j \uparrow}\right)-\frac{1}{2} L, \\
& {\left[\eta, \eta^{\dagger}\right]=-2 \eta^{z}, \quad\left[\eta, \eta^{z}\right]=\eta, \quad\left[\eta^{\dagger}, \eta^{z}\right]=-\eta^{\dagger} .}
\end{aligned}
$$

The global symmetry of the model (1) corresponding to these two $S U(2)$ symmetries is $S O(4)$, since half of the irreducible representations of $S U(2) \otimes S U(2)$ are excluded. The BA solution refers to the Hilbert subspace spanned by the lowest-weight states (LWSs) of both the spin and $\eta$-spin algebras. The subspace which is not associated with such a

solution is spanned by the energy eigenstates obtained by applying onto the LWSs one of the off-diagonal generators of the corresponding two algebras [25].

The charge and spin current operators of the model read [19],

$$
\begin{aligned}
& J^{\rho}=-e i t \sum_{\sigma} \sum_{j=1}^{L}\left(c_{j \sigma}^{\dagger} c_{j+1 \sigma}-c_{j+1 \sigma}^{\dagger} c_{j \sigma}\right) \\
& J^{\sigma z}=-\frac{1}{2} i t \sum_{\sigma} \sum_{j=1}^{L} \sigma\left(c_{j \sigma}^{\dagger} c_{j+1 \sigma}-c_{j+1 \sigma}^{\dagger} c_{j \sigma}\right) .
\end{aligned}
$$

In order to calculate the expectation values of the charge and spin current operators it is convenient to consider a uniform vector potential $A_{x} \vec{e}_{x}$, which modifies the hopping term along the chain by the usual Peierls phase factor, $t \rightarrow t \exp \left( \pm i \phi_{\sigma} / L\right)$. Following such a procedure, the Hamiltonian becomes,

$$
\begin{aligned}
\mathcal{H}= & -t \sum_{j, \sigma}\left(c_{j \sigma}^{\dagger} c_{j+1 \sigma} e^{i \phi_{\sigma} / L}+c_{j+1 \sigma}^{\dagger} c_{j \sigma} e^{-i \phi_{\sigma} / L}\right) \\
& +U \sum_{j}\left(n_{j, \uparrow}-1 / 2\right)\left(n_{j, \downarrow}-1 / 2\right) .
\end{aligned}
$$


For a given energy eigenstate $|m\rangle$, the charge and spin current expectation values $J_{m}^{\rho}=\left\langle m\left|J^{\rho}\right| m\right\rangle$ and $J_{m}^{\sigma_{z}}=\left\langle m\left|J^{\sigma_{z}}\right| m\right\rangle$, respectively, can be expressed as follows [26],

$$
\begin{array}{ll}
J_{m}^{\rho}=\left.\frac{d E_{m}(\phi)}{d(\phi / L)}\right|_{\phi=0} & \phi=\phi_{\uparrow}=\phi_{\downarrow}, \\
J_{m}^{\sigma_{z}}=\left.\frac{d E_{m}(\phi)}{d(\phi / L)}\right|_{\phi=0} & \phi=\phi_{\uparrow}=-\phi_{\downarrow} .
\end{array}
$$

The $\phi_{\sigma}>0$ Hamiltonian (4) remains integrable and can be diagonalized by means of coordinate BA [9]. One can introduce two generalized $S U(2)$ symmetries for the $\phi_{\sigma}>0$ case [27]. Since $2 \eta$ and $S$ remain good quantum numbers, one finds that the BA solution refers to the LWSs of both the $\eta$-spin and spin generalized algebras. Thus, all the energies of the tower of states such that $2 \eta>[L-N]$ and $2 S>\left[N_{\uparrow}-N_{\downarrow}\right]$ have the same energy as the corresponding LWSs. It follows that the BA numbers can be related to the values $\eta$ and $S$ of the states of each $\eta$-spin and spin tower, respectively. Since the studies of the ensuing section refers to both LWSs and non-LWSs, here we express the sum rules of the BA numbers in terms of the good quantum numbers $\eta$ and $S$. Moreover, we provide the simplified expressions in terms of the electronic numbers $N$ and $N_{\sigma}$ which correspond to the LWS of each tower only.

The solution of the Hamiltonian (4) by the BA leads to the following equations $[9,28]$,

$$
\begin{aligned}
& e^{i k_{j} L}=e^{i \phi_{\uparrow}} \prod_{\beta=1}^{M} \frac{\sin k_{j}-\Lambda_{\beta}+i u}{\sin k_{j}-\Lambda_{\beta}-i u}, \\
& e^{i\left(\phi_{\downarrow}-\phi_{\uparrow}\right)} \prod_{j=1}^{N_{c}} \frac{\Lambda_{\gamma}-\sin k_{j}+i u}{\Lambda_{\gamma}-\sin k_{j}-i u} \\
& =-\prod_{\beta=1}^{M} \frac{\Lambda_{\gamma}-\Lambda_{\beta}+i 2 u}{\Lambda_{\gamma}-\Lambda_{\beta}-i 2 u}
\end{aligned}
$$

Here and throughout this paper $u=U / 4 t$, the numbers $N_{c}$ and $M$ such that $0 \leq N_{c} \leq N$ and $0 \leq M \leq N_{\downarrow}$, respectively, are defined below, and $\Lambda$ is the spin rapidity [7]. Takahashi string hypothesis states that in addition to the real solutions for $\Lambda_{\gamma}$ and $k_{j}$, there are solutions involving complex $k_{j}$ and $\Lambda_{\gamma}$ values. The spin string $\Lambda$ s of legth $n$ is characterized by [7],

$$
\Lambda_{\gamma}^{n j}=\Lambda_{\gamma}^{n}+(n+1-2 j) i u . \quad j=1,2, \ldots, n .,
$$

where $\Lambda_{\gamma}^{n}$ is the real part of the complex number. The charge $k-\Lambda$ string of length $n$ includes $2 n k \mathrm{~s}$ and $n \Lambda \mathrm{s}$ such that,

$$
\begin{aligned}
& \Lambda_{\gamma}^{\prime n j}=\Lambda_{\gamma}^{\prime n}+(n+1-2 j) i u, \quad n=1,2, \ldots, n, \\
& k_{\gamma}^{1}=\pi-\sin ^{-1}\left(\Lambda_{\gamma}^{\prime n}+n i u\right), \\
& k_{\gamma}^{2}=\sin ^{-1}\left(\Lambda_{\gamma}^{\prime n}+(n-2) i u\right),
\end{aligned}
$$


Charge and Spin Transport in the One-dimensional Hubbard Model

$$
\begin{aligned}
& k_{\gamma}^{3}=\pi-k_{\gamma}^{2}, \\
& k_{\gamma}^{4}=\sin ^{-1}\left(\Lambda_{\gamma}^{\prime n}+(n-4) i u\right), \\
& k_{\gamma}^{5}=\pi-k_{\gamma}^{4}, \\
& \ldots, \\
& k_{\gamma}^{2 n-2}=\sin ^{-1}\left(\Lambda_{\gamma}^{\prime n}-(n-2) i u\right), \\
& k_{\gamma}^{2 n-1}=\pi-k_{\gamma}^{2 n-2}, \\
& k_{\gamma}^{2 n}=\pi-\sin ^{-1}\left(\Lambda_{\gamma}^{\prime n}-n i u\right) .
\end{aligned}
$$

By use of Eqs. (7) and (8) in Eqs. (6) we arrive to the following transcendental equations $[28,29]$,

$$
\begin{aligned}
& k_{j} L=2 \pi I_{j}+\phi_{\uparrow}-\sum_{n=1}^{\infty} \sum_{\beta=1}^{M_{n}} \theta\left(\frac{\sin k_{j}-\Lambda_{\beta}^{n}}{n u}\right)-\sum_{n=1}^{\infty} \sum_{\beta=1}^{M_{n}^{\prime}} \theta\left(\frac{\sin k_{j}-\Lambda_{\beta}^{\prime n}}{n u}\right) \\
& L\left(\sin ^{-1}\left(\Lambda_{\gamma}^{\prime n}+i n u\right)+\sin ^{-1}\left(\Lambda_{\gamma}^{\prime n}-i n u\right)\right)=2 \pi J_{\gamma}^{\prime n}-n\left(\phi_{\uparrow}+\phi_{\downarrow}\right) \\
& +\sum_{j=1}^{N-2 M^{\prime}} \theta\left(\frac{\Lambda_{\gamma}^{\prime n}-\sin k_{j}}{n u}\right)+\sum_{m, \beta} \Theta_{n m}\left(\frac{\Lambda_{\gamma}^{\prime n}-\Lambda_{\beta}^{\prime m}}{u}\right), \\
& \sum_{j=1}^{N-2 M^{\prime}} \theta\left(\frac{\Lambda_{\gamma}^{n}-\sin k_{j}}{n u}\right)=2 \pi J_{\gamma}^{n}+n\left(\phi_{\downarrow}-\phi_{\uparrow}\right)+\sum_{m, \beta} \Theta_{n m}\left(\frac{\Lambda_{\gamma}^{n}-\Lambda_{\beta}^{m}}{u}\right),
\end{aligned}
$$

where $\theta(x)=-2 \tan (x)$ and

$$
\begin{aligned}
\Theta_{n m}(x)= & \theta\left(\frac{x}{|n-m|}\right)+2 \theta\left(\frac{x}{|n-m|+2}\right)+\cdots+2 \theta\left(\frac{x}{n+m-2}\right) \\
& +\theta\left(\frac{x}{n+m}\right), \text { for }: n \neq m \\
= & 2 \theta\left(\frac{x}{2}\right)+2 \theta\left(\frac{x}{4}\right)+\cdots+2 \theta\left(\frac{x}{2 n-2}\right)+\theta\left(\frac{x}{2 n}\right), \\
& \text { for }: n=m .
\end{aligned}
$$

Here $\left\{I_{j}, J_{\gamma}^{\prime n}, J_{\gamma}^{n}\right\}$ are the actual quantum numbers whose values define the energy eigenstates and thus determine the energy and current spectra of the elementary excitations studied in the ensuing section. Following the notation of Takahashi [7], we introduce the numbers,

$$
M_{c}=\sum_{n} n M_{n}^{\prime}, \quad M_{s}=\sum_{n} n M_{n} .
$$

In these expressions $M_{n}$ and $M_{n}^{\prime}$ are the numbers of spin $\Lambda$ strings of length $n$ and charge $k-\Lambda$ strings of length $n$, respectively. The values of $N_{c}$ and $M$ are then uniquely defined by the following sum rules,

$$
N_{c}=L-2 \eta-2 M_{c}, \quad M=M_{c}+M_{s}=\frac{L}{2}-\eta-S,
$$

which for a LWS of the $\eta$-spin and algebras such that $2 \eta=[L-N]$ and $2 S=\left[N_{\uparrow}-N_{\downarrow}\right]$, respectively, simplify to,

$$
N_{c}=N-2 M_{c}, \quad M=N_{\downarrow} .
$$


The above quantum numbers $\left\{I_{j}, J_{\gamma}^{\prime n}, J_{\gamma}^{n}\right\}$ can be integers or half-odd integers (HOI) according to the following prescriptions: $I_{j}$ is integer (or HOI) if $\sum_{m}\left(M_{m}+M_{m}^{\prime}\right.$ ) is even (odd); $J_{\gamma}^{n}$ is integer (HOI) if $N_{c}-M_{n}$ is odd (even); $J_{\gamma}^{\prime n}$ is integer (HOI) if $L-\left(N_{c}-M_{n}^{\prime}\right)$ is odd (even). They have values in the following ranges,

$$
\begin{aligned}
& I_{j}<\frac{L}{2}, \\
& J_{\gamma}^{\prime n}<\frac{1}{2}\left(L-N+2 M_{c}-\sum_{m=1}^{\infty} t_{n m} M_{m}^{\prime}\right), \\
& J_{\gamma}^{n}<\frac{1}{2}\left(N-2 M_{c}-\sum_{m=1}^{\infty} t_{n m} M_{m}\right),
\end{aligned}
$$

where $t_{n m}=2 \min (n, m)-\delta_{n m}$.

The energy and momentum spectra are given in terms of the BA quantum numbers as follows,

$$
\begin{aligned}
E= & -\sum_{j=1}^{N_{c}} 2 t \cos k_{j}+\sum_{n, \alpha} 4 t \mathcal{R} \sqrt{1-\left(\lambda_{\alpha}^{\prime n}-i n u\right)^{2}} \\
& -\frac{U}{2}\left(N_{c}+2 M_{c}-\frac{1}{2}\right), \\
P= & \frac{2 \pi}{L}\left(\sum_{j} I_{j}+\sum_{n, \alpha} J_{\alpha}^{n}\right)+\sum_{n, \alpha}\left(\pi-\frac{2 \pi}{L} J_{\alpha}^{\prime n}\right) \\
& +\pi\left(M_{c}+\eta-\frac{1}{2}[L-N]\right),
\end{aligned}
$$

where $\mathcal{R}$ refers to the real part and $\pi\left(M_{c}+\eta-\frac{1}{2}[L-N]\right)$ simplifies to $\pi M_{c}$ for a LWS of the $\eta$-spin algebra.

All energy eigenstates associated with the BA solution are described by different occupancy configurations of the quantum numbers appearing in the system of coupled equations given in Eq. (9). For example, for the ground state there is no complex solution for Eqs. (6) and $I_{j}$ and $J_{\gamma}$ are successive numbers centered around zero. Thus, the quantum number occupancy configuration for even $N_{c}=N=L$ and odd $N_{c} / 2$ corresponds to,

$$
\begin{aligned}
& I_{j}=-\frac{N-1}{2},-\frac{N-3}{2}, \cdots, \frac{N-1}{2}, \\
& J_{\gamma}=-\frac{M-1}{2},-\frac{M-3}{2}, \cdots, \frac{M-1}{2} .
\end{aligned}
$$

In some of the figures presented in the ensuing section we measure the energy relative to the ground-state energy. Such a choice corresponds to the following general energy spectrum,

$$
\begin{aligned}
E_{0}= & -\sum_{j=1}^{N_{c}} 2 t \cos k_{j}+\sum_{n, \alpha} 4 t \mathcal{R} \sqrt{1-\left(\lambda_{\alpha}^{\prime n}-i n u\right)^{2}} \\
& -\frac{U}{2}\left[N_{c}+2 M_{c}-\frac{1}{2}\right]-2 \mu\left(N_{a}-N\right)-2 \mu_{0} H\left(N_{\uparrow}-N_{\downarrow}\right),
\end{aligned}
$$


where $\mu$ is the chemical potential, $\mu_{0}$ the Bohr magneton, and $H$ the magnetic field.

Although the Bethe-ansatz equations (9) refer to $L>>1$, we have used these equations in the numerical study of finite- $L$ chains and obtained results for several quantities in excellent numerical agreement with the known exact values. Thus, in the ensuing section we use these equations to derive numerically the charge and spin currents carried by the elementary excitations for finite values of $L$, as well as their energy spectra. The obtained results are a good approximation for the corresponding current spectra of the $L>>1$ quantum problem which the equations (9) refer to.

\section{Current spectra for a finite-size system}

\subsection{The case of half-filling}

The zero-magnetization and half-filling ground state is both a spin and $\eta$-spin singlet. Thus, the simplest elementary excitations are spin-triplet excitations such that $(\eta=$ $0, S=1)$, spin-singlet excitations such that $(\eta=0, S=0)$ whose energy spectrum is degenerated to that of the spin-triplet excitations, charge $\eta$-spin-triplet excitations such that $(\eta=1, S=0)$, and charge $\eta$-spin-singlet excitations such that $(\eta=0, S=0)$ whose energy spectrum is degenerated to that of the charge $\eta$-spin-triplet excitations. The energy spectra of these four elementary excitations have been previously studied [30]. For a comparison, we evaluate the energy spectrum and the charge and spin currents of all the energy eigenstates corresponding to the above four types of halffilling elementary excitations. The energies considered in this subsection correspond to the general energy spectrum provided in Eq. (15).

The half-filling and zero-magnetization ground state considered in this subsection is characterized by charge and spin distributions given by,

$$
\begin{aligned}
& \rho_{0}(k)=\frac{1}{2 \pi}+\frac{\cos k}{\pi} \int_{0}^{\infty} \frac{J_{0}(p) \cos (p \sin k)}{1+e^{u|p| / 2}} d p, \\
& \sigma_{0}(\lambda)=\frac{1}{8 \pi u} \int_{-\pi}^{\pi} \operatorname{sech}\left[\frac{2 \pi}{u}(\lambda-\sin k)\right] d k,
\end{aligned}
$$

where $J_{0}$ is the Bessel function of zero order. Following standard BA procedures, the evaluation of the energy, charge-current, and spin-current spectra studied below involves the use of corresponding distributions for the elementary excitations.

Spin-triplet excitations. Such elementary excitations are obtained by introducing "holes" in the spin distribution of the numbers $J_{\gamma}$ relative to the ground-state occupancy configuration. For each value of the excitation momentum and energy there is a spin tower of three $S=1$ states, differing in the spin projections $0, \pm 1$, but all having the same values,

$$
N_{c}=N=L, \quad M=L / 2-1,
$$

and

$$
I_{j}=-\frac{N-2}{2},-\frac{N-4}{2}, \cdots, \frac{N}{2}
$$


Charge and Spin Transport in the One-dimensional Hubbard Model

$$
J_{\gamma}^{1}=-\frac{M+1}{2},-\frac{M-3}{2}, \cdots, \frac{M+1}{2}
$$

for the BA numbers. Hence, there are two holes $\lambda_{1}^{h}$ and $\lambda_{2}^{h}$ in the spin distribution. The BA equations become,

$$
\begin{aligned}
& k_{j} L=2 \pi I_{j}+\phi_{\uparrow}-2 \sum_{\beta=1}^{L / 2-1} \tan ^{-1} \frac{\sin k_{j}-\lambda_{\beta}}{u} \\
& \sum_{j=1}^{N} 2 \tan ^{-1} \frac{\lambda_{\gamma}-\sin k_{j}}{u}=2 \pi J_{\gamma}+\left(\phi_{\downarrow}-\phi_{\uparrow}\right) \\
& +2 \sum_{\beta=1}^{L / 2-1} \tan ^{-1} \frac{\lambda_{\gamma}-\lambda_{\beta}}{2 u} .
\end{aligned}
$$

Following the usual procedures of BA [7, 28], one then introduces the following charge and spin distributions,

$$
\begin{aligned}
\rho(k)=\frac{1}{2 \pi}+ & \cos k \int K_{1}(\sin k-\lambda) \sigma(\lambda) d \lambda, \\
\sigma(\lambda)+\sigma^{h}(\lambda)= & \int K_{1}(\lambda-\sin k) \rho(k) d k \\
& -\int K_{2}\left(\lambda-\lambda^{\prime}\right) \sigma\left(\lambda^{\prime}\right) d \lambda^{\prime},
\end{aligned}
$$

where $\sigma^{h}(\lambda)=\left[\delta\left(\lambda-\lambda_{1}^{h}\right)+\delta\left(\lambda-\lambda_{2}^{h}\right)\right] / L$ and $K_{n}(x)=n u /\left[\pi\left(n^{2} u^{2}+x^{2}\right)\right]$. In the presence of the flux the momentum deviation corresponding to this elementary excitation is given by,

$$
\begin{aligned}
& \triangle k_{j} L=\phi_{\uparrow} \\
& -2 \pi \sum_{\beta=1}^{L / 2-1} K_{1}\left(\sin k_{j}-\lambda_{\beta}\right)\left(\cos k_{j} \triangle k_{j}-\triangle \lambda_{\beta}\right), \\
& 2 \pi \sum_{j=1}^{N} K_{1}\left(\lambda_{\gamma}-\sin k_{j}\right)\left(\Delta \lambda_{\gamma}-\cos k_{j} \triangle k_{j}\right)=\left(\phi_{\downarrow}-\phi_{\uparrow}\right) \\
& +2 \pi \sum_{\beta=1}^{L / 2-1} K_{2}\left(\lambda_{\gamma}-\lambda_{\beta}\right)\left(\triangle \lambda_{\gamma}-\triangle \lambda_{\beta}\right) .
\end{aligned}
$$

Use of Eq. (22) then yields,

$$
\begin{aligned}
& \triangle k \rho(k)=\frac{\phi_{\uparrow}}{2 \pi L}+\int K_{1}(\sin k-\lambda) \triangle \lambda \sigma(\lambda) d \lambda, \\
& \triangle \lambda\left[\sigma(\lambda)+\sigma^{h}(\lambda)\right]=\frac{\phi_{\downarrow}-\phi_{\uparrow}}{2 \pi L}-\int K_{2}\left(\lambda-\lambda^{\prime}\right) \Delta \lambda^{\prime} \sigma\left(\lambda^{\prime}\right) d \lambda^{\prime} \\
& \quad+\int K_{1}(\lambda-\sin k) \cos k \Delta k \rho(k) d k .
\end{aligned}
$$

The corresponding energy deviation is given by

$$
\triangle E(\phi)=2 t L \int \sin k \rho(k) \triangle k d k
$$



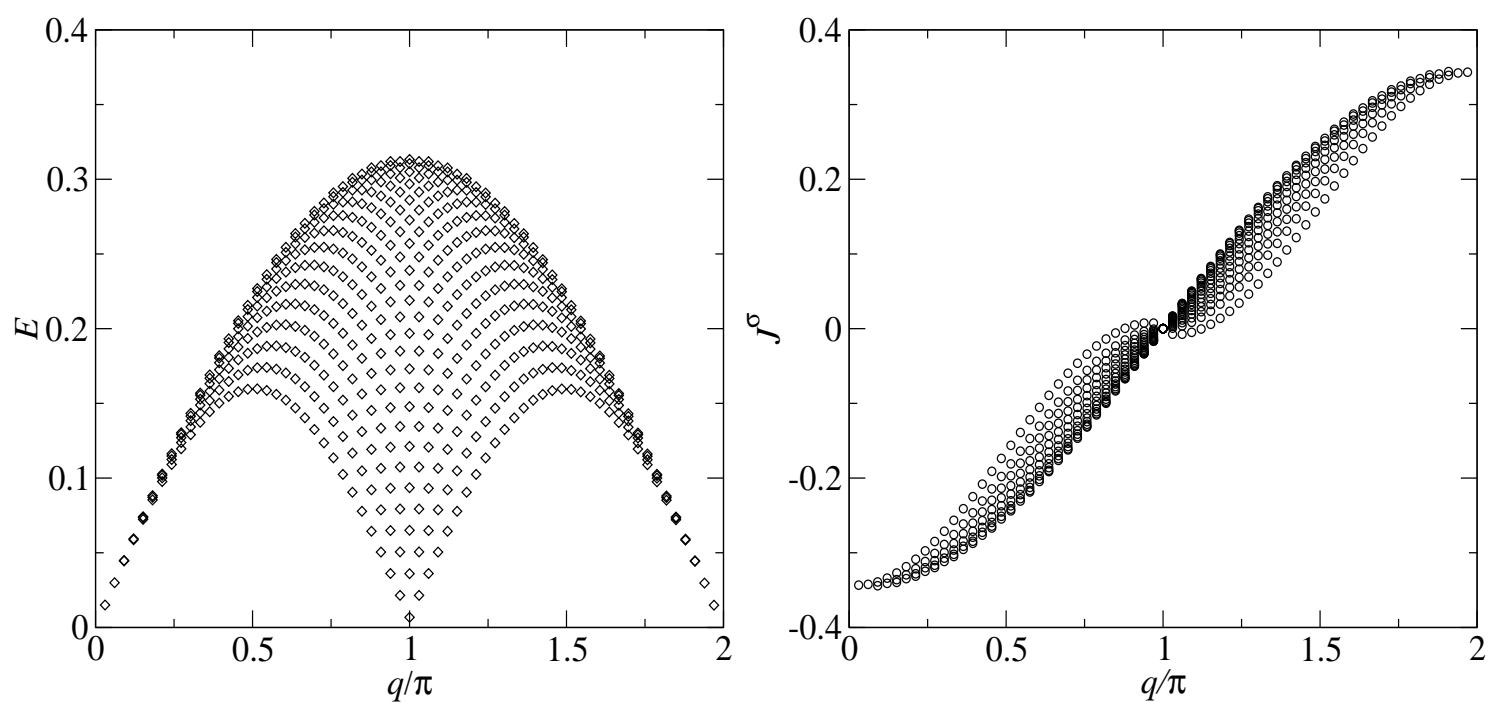

Figure 1. The half-filling energy spectrum (left) and spin current spectrum (right) of the spin triplet excitations for $u=10, N=L=66$, and $M=33$.

Our next task is the solution of the equations given in (24). Inserting the result obtained for $\Delta k \rho(k)$ into Eq. (25) we find,

$$
\Delta E=-\frac{t\left(\phi_{\downarrow}-\phi_{\uparrow}\right)}{8 \pi u L} \int d k \sin k\left[\frac{\operatorname{sech}\left[\frac{\pi}{2 u}\left(\sin k-\lambda_{1}^{h}\right)\right]}{\sigma_{0}\left(\lambda_{1}^{h}\right)}-\frac{\operatorname{sech}\left[\frac{\pi}{2 u}\left(\sin k-\lambda_{2}^{h}\right)\right]}{\sigma_{0}\left(\lambda_{2}^{h}\right)}\right],
$$

where we have used the relation $\Delta \lambda \simeq\left(\phi_{\downarrow}-\phi_{\uparrow}\right) /\left(4 \pi L \sigma_{0}(\lambda)\right.$, which was obtained by Fourier transformation. It then follows that when $\phi_{\downarrow}=\phi_{\uparrow}$ the charge current defined by Eq. (5) vanishes.

In general case, $\lambda^{h}>1$, Eq. (26) can be solved approximately with the result,

$$
J^{\sigma} \approx \frac{t \pi}{2 u}\left[\tanh \left(\frac{\pi \lambda_{1}^{h}}{2 u}\right)+\tanh \left(\frac{\pi \lambda_{2}^{h}}{2 u}\right)\right] .
$$

Since the momentum carried by the spin elementary excitation has the form $q=$ $2 \tan ^{-1} e^{-\lambda / 2 u}-\frac{\pi}{2}$ and the quantum number of the charge part changes from half-integer to integer, what generates a momentum shift $\pi$, the spin-current spectrum has the following form, as was also observed in the antiferromagnetic Heisenberg model [31],

$$
\begin{aligned}
J^{\sigma} & =\frac{t \pi}{2 u}\left[\sin q_{1}+\sin q_{2}\right], \\
q & =\pi+q_{1}+q_{2}, \quad q_{1}, q_{2} \in\left[-\frac{\pi}{2}, \frac{\pi}{2}\right] .
\end{aligned}
$$

Here $q_{1}$ and $q_{2}$ are the momenta of the two spin-distribution holes and $q$ denotes the total excitation momentum. In figures 1 and 2 we plot the energy and spin-current spectra of the spin-triplet excitations for $u=10$ and $u=1$, respectively. Such spectra were obtained by solving numerically the BA equations. 

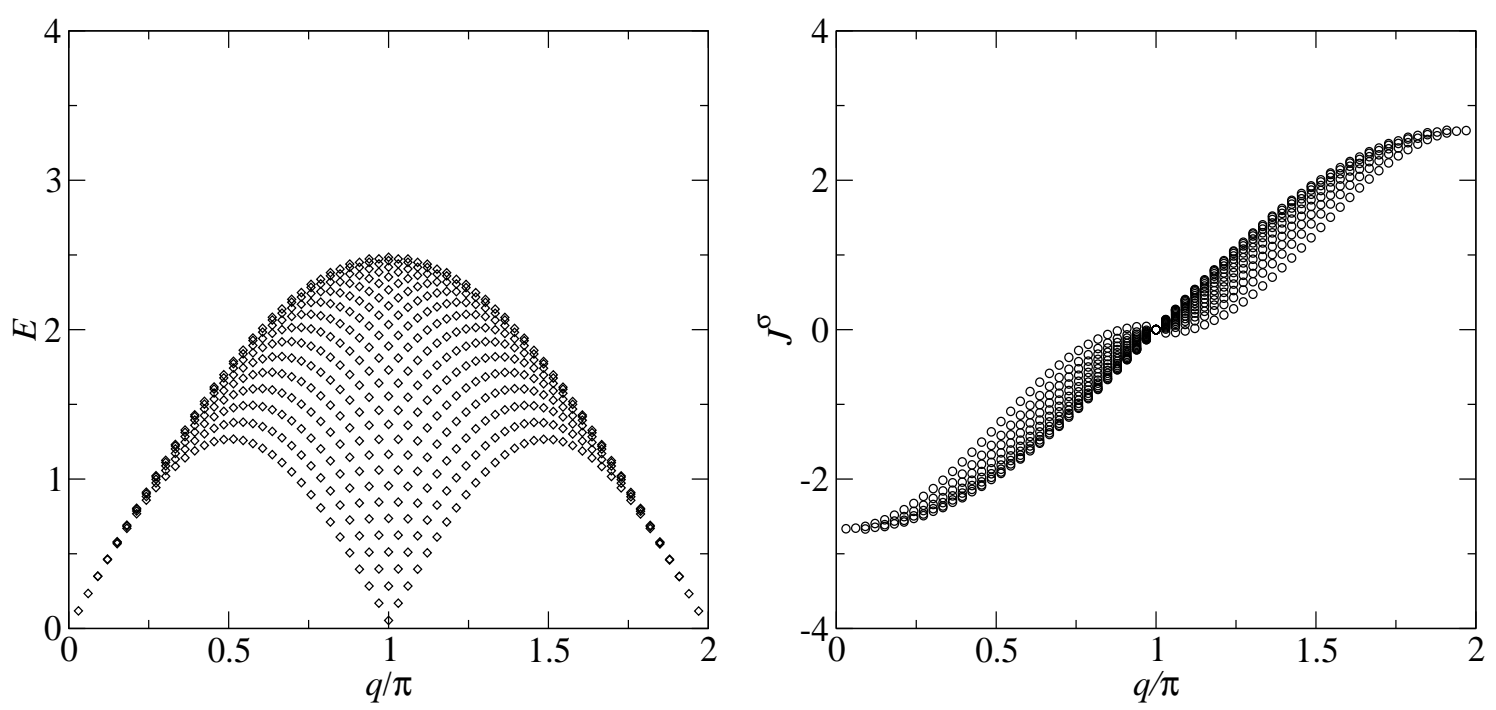

Figure 2. The half-filling energy spectrum (left) and spin current spectrum (right) of the spin triplet excitations for $u=1, N=L=66$, and $M=33$.

The group velocity $v^{\sigma(\rho)}(q)$ and the effective spin (charge) $e^{\sigma(\rho)}$ are defined as,

$$
v^{\sigma(\rho)}(q)=\frac{d E^{\sigma(\rho)}(q)}{d q}, \quad e^{\sigma(\rho)}=e \frac{J^{\sigma(\rho)}(q)}{v^{\sigma(\rho)}(q)} .
$$

In this equation $\sigma$ and $\rho$ denote the spin current and charge current, respectively, and $e=-1$ and $e=1 / 2$ for the charge and spin cases. Thus, the group velocity of a single spin-distribution hole reads,

$$
v^{\sigma}(q)=-\frac{t \pi}{2 u} \sin q,
$$

whereas the corresponding effective spin is given by,

$$
e^{\sigma}=-\frac{1}{2}
$$

Note that the total spin current is positive.

Spin-singlet excitations. The second type of elementary spin excitations studied here corresponds to the spin-singlet excitations whose energy spectrum is degenerated with that of the spin-triplet excitations considered above. Such excitations have a spin string of length one. Thus, the BA numbers are given by,

$$
M_{1}=M-2, \quad M_{2}=1,
$$

and

$$
\begin{aligned}
& I_{j}=-\frac{N-2}{2},-\frac{N-4}{2}, \cdots, \frac{N}{2}, \\
& J_{\gamma}^{1}=-\frac{M-1}{2},-\frac{M-3}{2}, \cdots, \frac{M-1}{2} \\
& J_{0}^{2}=0 .
\end{aligned}
$$



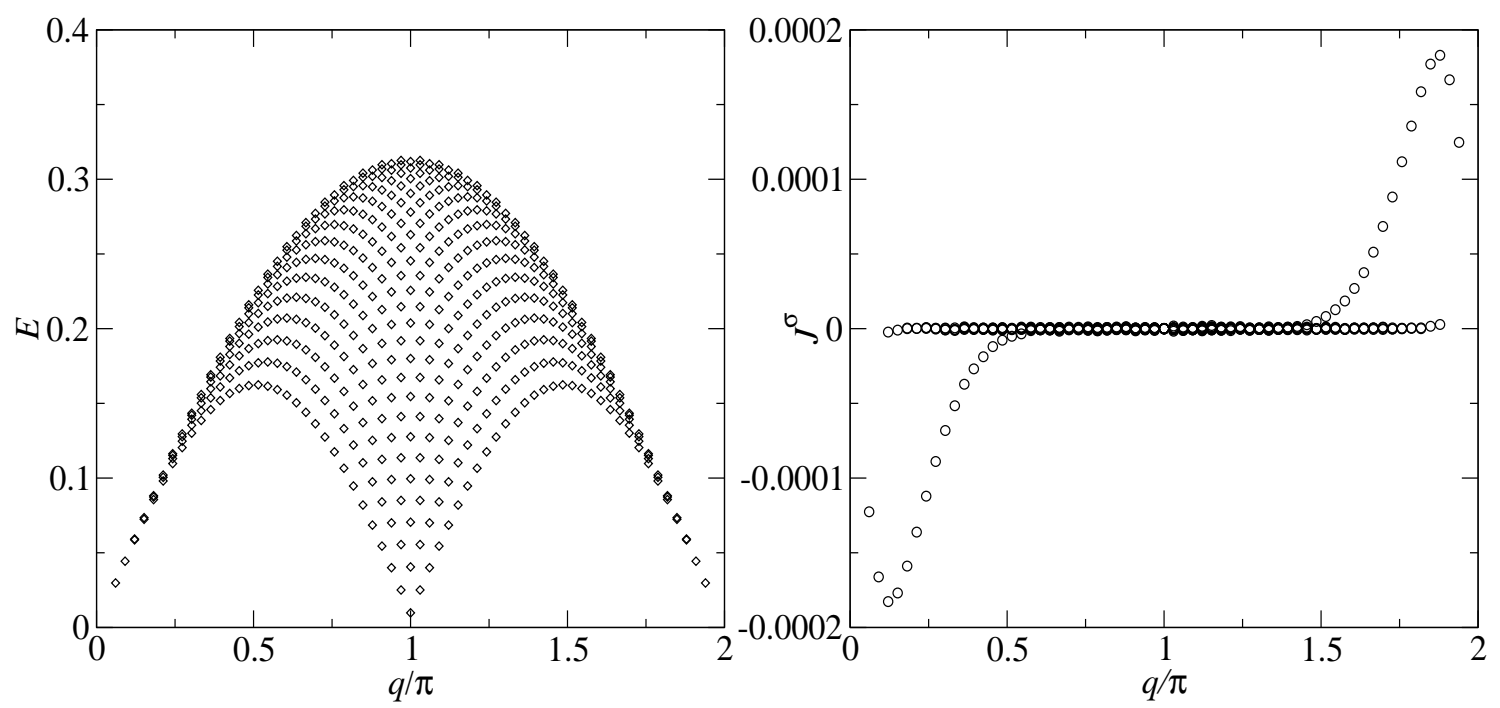

Figure 3. The half-filling energy spectrum (left) and spin current spectrum (right) of the spin-singlet excitations for $u=10, N=L=66$, and $M=33$.
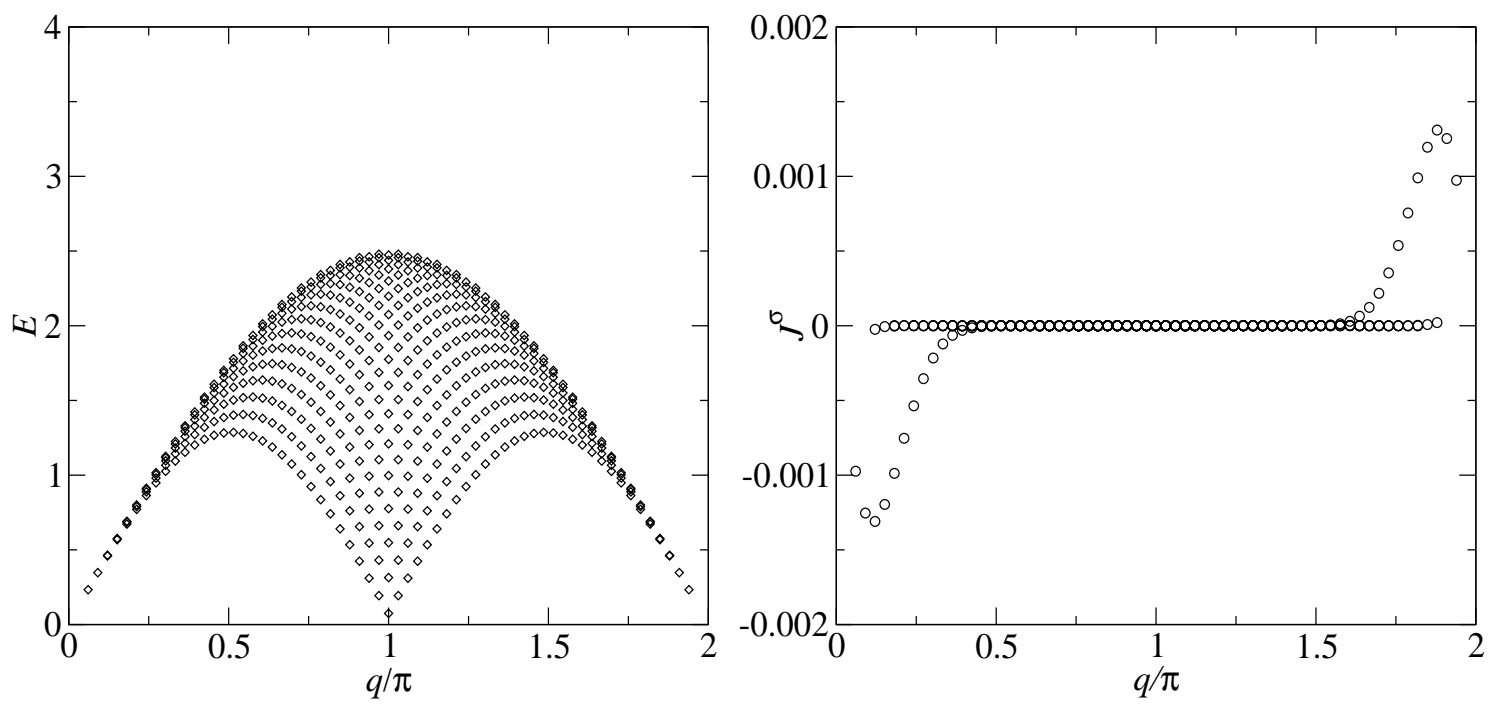

Figure 4. The half-filling energy spectrum (left) and spin current spectrum (right) of the spin-singlet excitations for $u=1, N=L=66$, and $M=33$.

It follows that there are again two holes in the spin distribution, $\lambda_{1}^{h}$ and $\lambda_{2}^{h}$. The BA equations are given by,

$$
\begin{aligned}
k_{j} L & =2 \pi I_{j}+\phi_{\uparrow}-2 \sum_{\beta=1}^{L / 2-2} \tan ^{-1} \frac{\sin k_{j}-\lambda_{\beta}}{u} \\
& -2 \tan ^{-1} \frac{\sin k_{j}-\Lambda}{2 u}
\end{aligned}
$$



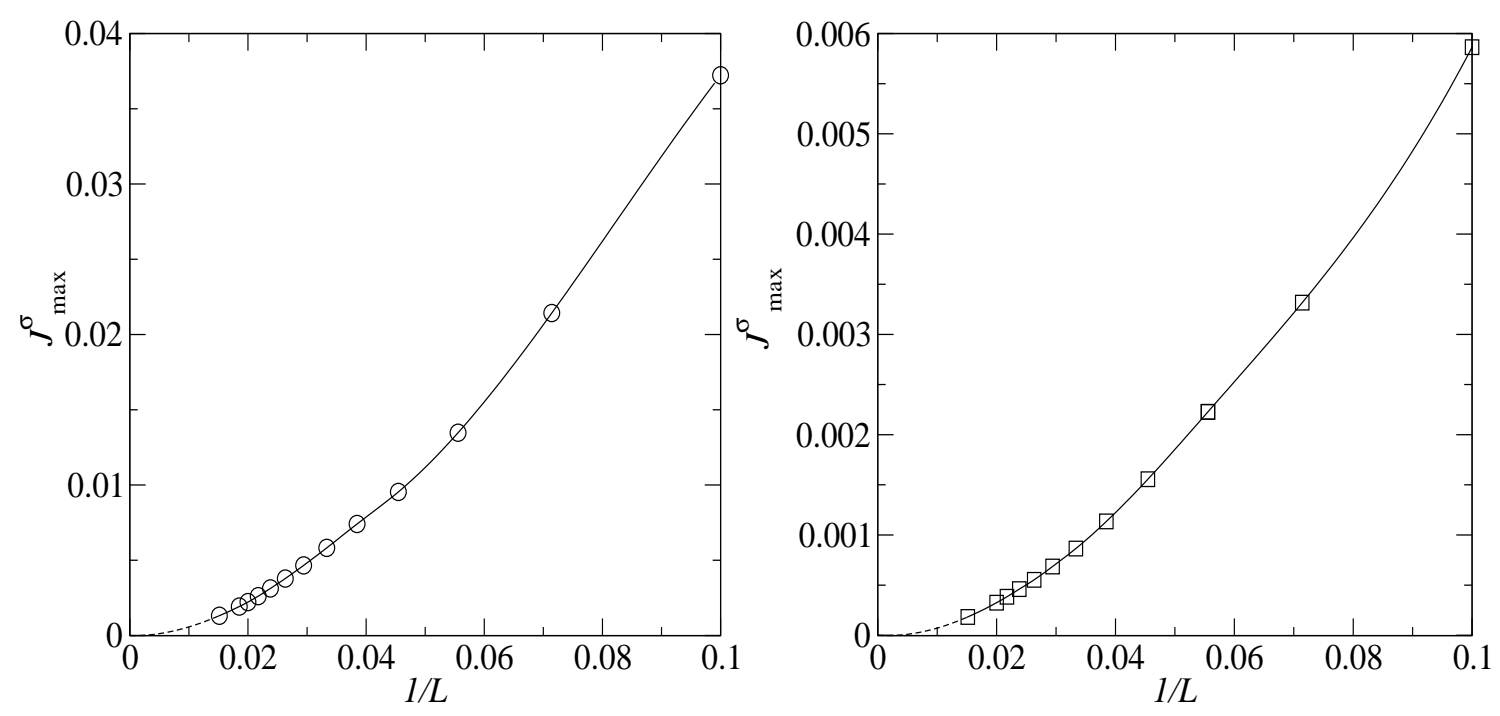

Figure 5. Scaling analysis of the maximum current carried by spin-singlet excitations for $u=1$ (left) and $u=10$ (right) respectively.

$$
\begin{aligned}
& 2 \sum_{j=1}^{N} \tan ^{-1} \frac{\lambda_{\gamma}-\sin k_{j}}{u}=2 \pi J_{\gamma}+\left(\phi_{\downarrow}-\phi_{\uparrow}\right) \\
& \quad+2 \sum_{\beta=1}^{L / 2-2} \tan ^{-1} \frac{\lambda_{\gamma}-\lambda_{\beta}}{2 u} \\
& \quad+2 \tan ^{-1} \frac{\lambda_{\gamma}-\Lambda}{u}+2 \tan ^{-1} \frac{\lambda_{\gamma}-\Lambda}{3 u} \\
& 2 \sum_{j=1}^{N} \tan ^{-1} \frac{\Lambda-\sin k_{j}}{2 u}=2 \pi J_{1}^{(2)}(=0)+2\left(\phi_{\downarrow}-\phi_{\uparrow}\right) \\
& \quad+2 \sum_{\beta}^{L / 2-2}\left[\tan ^{-1} \frac{\Lambda-\lambda_{\beta}}{u}+\tan ^{-1} \frac{\Lambda-\lambda_{\beta}}{3 u}\right],
\end{aligned}
$$

where $\Lambda$ denotes the rapidity of the spin string excitation of length two. The deviations of the charge and spin distributions are such that,

$$
\begin{aligned}
& \triangle k \rho(k)=\frac{\phi_{\uparrow}}{2 \pi L}+\int K_{1}(\sin k-\lambda) \Delta \lambda \sigma(\lambda) d \lambda \\
& +\frac{1}{L} K_{2}(\sin k-\Lambda) \Delta \Lambda \\
& \triangle \lambda\left[\sigma(\lambda)+\sigma^{h}(\lambda)\right]=\frac{\phi_{\downarrow}-\phi_{\uparrow}}{2 \pi L} \\
& \quad-\int K_{2}\left(\lambda-\lambda^{\prime}\right) \Delta \lambda^{\prime} \sigma\left(\lambda^{\prime}\right) d \lambda^{\prime} \\
& +\int \cos k \Delta k \rho(k) K_{1}(\lambda-\sin k) d k \\
& \quad-\frac{1}{L}\left[K_{1}(\lambda-\Lambda)+K_{3}(\lambda-\Lambda)\right] \Delta \Lambda
\end{aligned}
$$


Charge and Spin Transport in the One-dimensional Hubbard Model

$$
\begin{aligned}
& \Delta \Lambda \sigma_{2}(\Lambda)=\frac{\phi_{\downarrow}-\phi_{\uparrow}}{\pi L} \\
& \quad-\int\left[K_{1}(\Lambda-\lambda)+K_{3}(\Lambda-\lambda)\right] \Delta \lambda \sigma(\lambda) d \lambda \\
& \quad+\int \cos k K_{2}(\Lambda-\sin k) \Delta k \rho(k) d k \\
& \quad-\int\left[2 K_{2}\left(\Lambda-\Lambda^{\prime}\right)+K_{4}\left(\Lambda-\Lambda^{\prime}\right)\right] \Delta \Lambda \sigma_{2} d \Lambda .
\end{aligned}
$$

Here $\sigma_{2}\left(\Lambda^{\prime}\right)=\delta\left(\Lambda^{\prime}-\Lambda\right) / L$ and $\sigma^{h}(\lambda)=\left[\delta\left(\lambda-\lambda_{1}^{h}\right)+\delta\left(\lambda-\lambda_{1}^{h}\right)\right] / L$. Integrations in the variables $k, \lambda, \Lambda$ lead to,

$$
\begin{aligned}
& \int \cos k \Delta k \rho(k)=0 \\
& \int \Delta \lambda \sigma(\lambda) d \lambda+\frac{1}{L} \Delta \Lambda=\int \frac{\phi_{\downarrow}-\phi_{\uparrow}}{4 \pi L} d \lambda-\int \Delta \lambda \sigma^{h}(\lambda) d \lambda, \\
& \frac{1}{L} \Delta \Lambda+\frac{1}{2} \int \Delta \lambda \sigma(\lambda) d \lambda=\int \frac{\phi_{\downarrow}-\phi_{\uparrow}}{4 \pi L} d \lambda .
\end{aligned}
$$

Finally, the deviation associated with the spin string excitation of length two has the form,

$$
\Delta \Lambda=\left(\Delta \lambda_{1}^{h}+\Delta \lambda_{2}^{h}\right)+\int \frac{\phi_{\downarrow}-\phi_{\uparrow}}{4 \pi} d \lambda,
$$

where the second term on the right-hand side can be omitted because it does not contribute to the spin current. Use of this expression in the first equation of (35) leads to,

$$
\triangle k \rho(k) \approx \frac{\phi_{\uparrow}}{2 \pi L}+\int K_{1}(\sin k-\lambda) \triangle \lambda\left[\sigma+\sigma^{h}\right] d \lambda .
$$

Since $\sigma_{0}(\lambda) \approx \sigma(\lambda)+\sigma^{h}(\lambda)$, by means of the same procedure already used for spin-triplet excitation, we find that to first order the energy deviation induced by the external flux vanishes. Thus, both the charge and spin currents carried by this type of elementary excitation vanish.

In figures 3 and 4 we plot the energy and spin current spectra of the spin-singlet excitations for $u=10$ and $u=1$, respectively. The two small features of the spincurrent spectrum result from finite-size effects and disapear in the thermodynamic limit, as shown in figure 5 .

We emphasize that although the group velocity of the two spin-distribution holes of the spin-singlet excitations is finite, the corresponding effective spin vanishes.

Charge $\eta$-spin-triplet excitations. For each value of the excitation momentum and energy there are three types of such $\eta=1$ elementary excitations, which correspond to the three values $0, \pm 1$ for the $\eta$-spin projection. All these excitations have again the same BA numbers,

$$
N=L-2, \quad M=N / 2,
$$



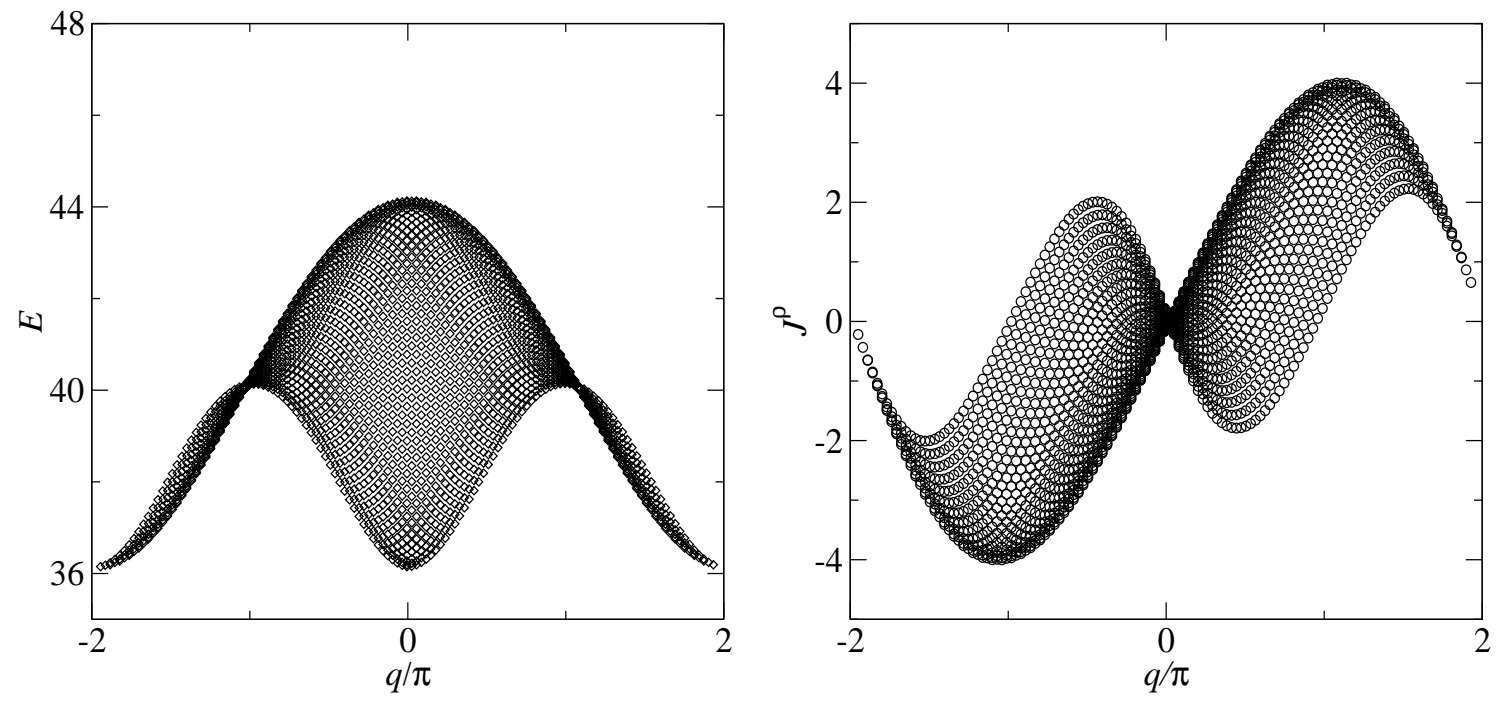

Figure 6. The half-filling energy spectrum (left) and charge-current spectrum (right) of the charge $\eta$-spin triplet excitation for $u=10, N=L=46$, and $M=23$.
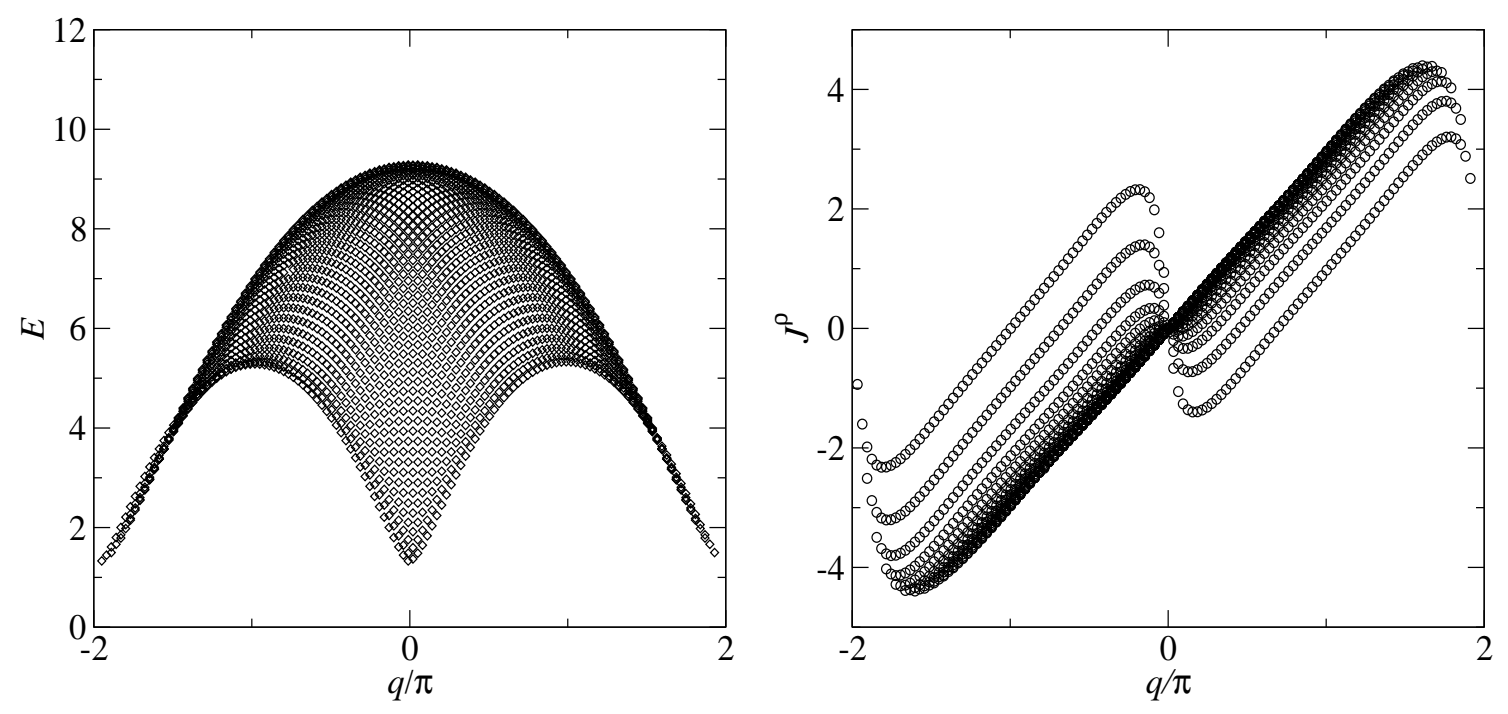

Figure 7. The half-filling energy spectrum (left) and charge-current spectrum (right) of the charge $\eta$-spin triplet excitation for $u=1, N=L=46$, and $M=23$.

and

$$
\begin{aligned}
& I_{j}=-\frac{L}{2}, \cdots, \frac{L}{2}-1, \\
& J_{\gamma}=-\frac{M-1}{2}, \cdots, \frac{M-1}{2} .
\end{aligned}
$$

There are two holes $k_{1}^{h}$ and $k_{2}^{h}$ in the charge distribution such that,

$$
\rho^{h}(k)=\frac{1}{L}\left[\delta\left(k-k_{1}^{h}\right)+\delta\left(k-k_{2}^{h}\right)\right] .
$$


Thus, the distributions $\rho(k)$ and $\sigma(\lambda)$ satisfy the following equations,

$$
\begin{aligned}
\rho(k)+ & \rho^{h}(k)=\frac{1}{2 \pi}+\cos k \int K_{1}(\sin k-\lambda) \sigma(\lambda) d \lambda \\
\sigma(\lambda)= & \int K_{1}(\lambda-\sin k) \rho(k) \\
& -\int K_{2}\left(\lambda-\lambda^{\prime}\right) \sigma\left(\lambda^{\prime}\right) d \lambda^{\prime} .
\end{aligned}
$$

The energy deviation corresponding to the two charge-distribution holes read,

$$
\Delta E(\phi)=-2 t\left[\sin k_{1}^{h} \Delta k_{1}^{h}+\sin k_{2}^{h} \Delta k_{2}^{h}\right],
$$

where the momentum deviation $\Delta k$ is approximately given by,

$$
\Delta k \approx \frac{\phi_{\uparrow}}{2 \pi L \rho_{0}(k)} .
$$

It follows that the charge current spectrum is such that,

$$
J^{\rho}=-2 t\left[\frac{\sin k_{1}^{h}}{\rho_{0}\left(k_{1}^{h}\right)}+\frac{\sin k_{2}^{h}}{\rho_{0}\left(k_{2}^{h}\right)}\right],
$$

while for $k^{h}=-k$ it reads,

$$
\begin{aligned}
J^{\rho} & =2 t\left[\frac{\sin k_{1}}{\rho_{0}\left(k_{1}\right)}+\frac{\sin k_{2}}{\rho_{0}\left(k_{2}\right)}\right], \\
k & =k_{1}+k_{2} .
\end{aligned}
$$

In turn, the spin current vanishes. In the strong coupling limit, $u=U / 4 t>>1$, one has that $\rho_{0}(k) \simeq 1 / 2 \pi$ and thus the spectrum simplifies to,

$$
J^{\rho} \propto \sin k_{1}+\sin k_{2} .
$$

In this case the corresponding energy spectrum can be expressed as the sum of three cosine functions, in addition to the energy gap. Hence, the velocity of a single chargedistribution hole simplifies to $v^{\rho}=-2 t \sin k_{h}$, whereas the effective charge is $-e$. This corresponds to a positive current in units of $e$.

Again, we used the BA equations to calculate the energy, charge-current, and spincurrent spectra for $u=10$ and $u=1$. (The spin current vanishes for the charge $\eta$-spintriplet states considered here.) The energy and charge-current spectra are plotted in Figs. 6 and 7 for $u=10$ and $u=1$, respectively. Note that the charge-current spectra features have a stronger linear character for $u=1$ than for $u=10$. We interpret this effect as due to the weak-coupling peak in the charge distribution as a function of the momentum $k$. When a hole is created away from zero momentum it is less affected by the other charges, leading to $J^{\rho} \propto q$.

Charge $\eta$-spin-singlet excitations. These $\eta=0$ elementary excitations are those whose energy spectrum is degenerated with that of the charge $\eta$-spin-triplet excitations considered above. Such $\eta$-spin-singlet excitations contain one charge string of length one. The BA numbers are then given by,

$$
M_{1}=\frac{N}{2}-1, \quad M_{1}^{\prime}=1,
$$



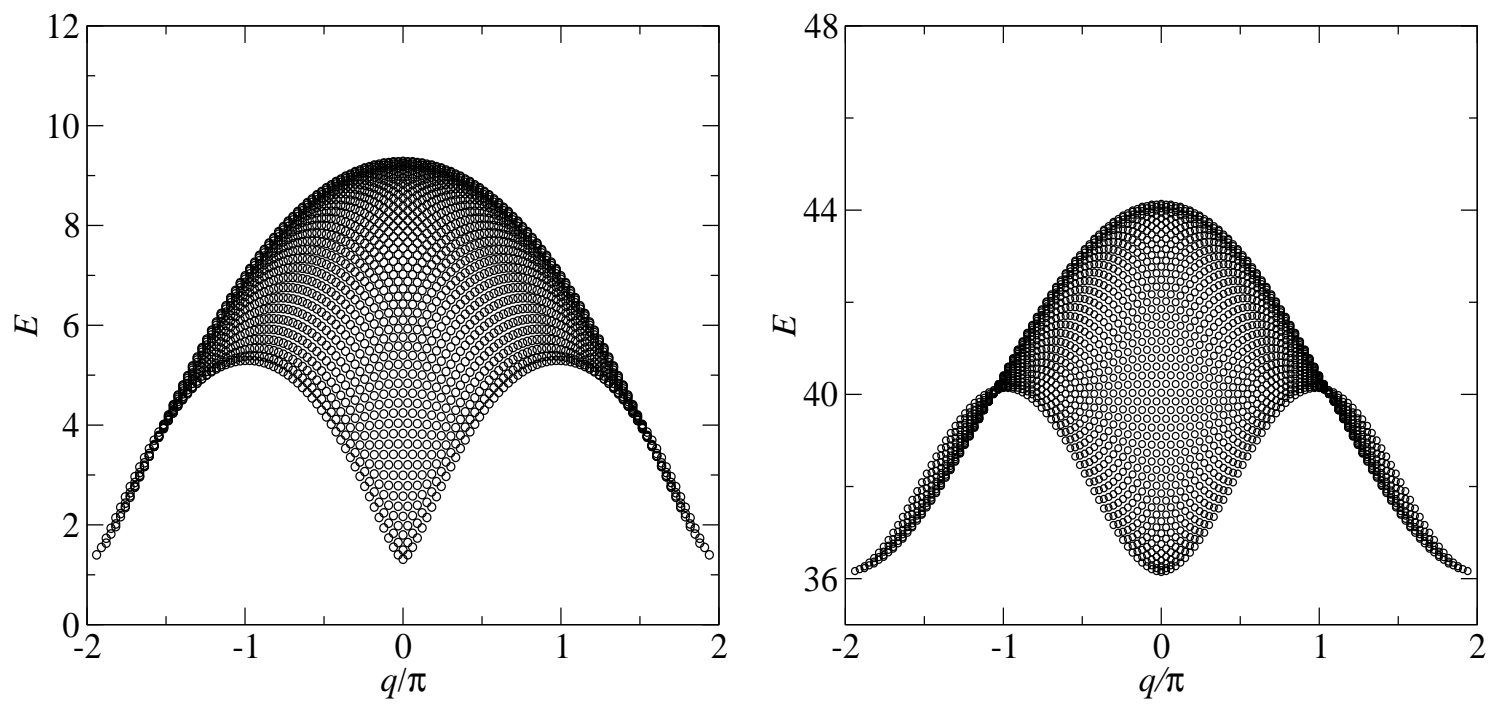

Figure 8. The half-filling energy spectra of charge $\eta$-spin singlet excitation for the strong coupling $u=1$ (left), and weak coupling $u=10$ (right).

and

$$
\begin{aligned}
I_{j} & =-\frac{N-1}{2}, \cdots, \frac{N-1}{2}, \\
J_{\gamma} & =-\frac{M_{1}-1}{2},-\frac{M_{1}-3}{2}, \cdots, \frac{M_{1}-1}{2} \\
J_{1}^{\prime} & =0 .
\end{aligned}
$$

These excitations involve two holes $k_{1}^{h}$ and $k_{2}^{h}$ in the charge distribution. The BA equations are such that,

$$
\begin{aligned}
& k_{j} L=2 \pi I_{j}+\phi_{\uparrow}-\sum_{\beta=1}^{L / 2-1} 2 \tan ^{-1} \frac{\sin k_{j}-\lambda_{\beta}}{u} \\
& -2 \tan ^{-1} \frac{\sin k_{j}-\Lambda}{u} \\
& \sum_{j=1}^{N-2} 2 \tan ^{-1} \frac{\lambda_{\gamma}-\sin k_{j}}{u}=2 \pi J_{\gamma}+\left(\phi_{\downarrow}-\phi_{\uparrow}\right) \\
& +\sum_{\beta=1}^{N / 2-1} 2 \tan ^{-1} \frac{\lambda_{\gamma}-\lambda_{\beta}}{2 u}, \\
& L\left[\sin ^{-1}(\Lambda+i u)+\sin ^{-1}(\Lambda-i u)\right] \\
& =2 \pi J_{1}^{\prime 1}-\left(\phi_{\uparrow}+\phi_{\downarrow}\right)+2 \sum_{j=1}^{L-2} \tan ^{-1} \frac{\Lambda-\sin k_{j}}{u},
\end{aligned}
$$

where $\Lambda$ is the rapidity involved in the charge string of length-one. Moreover, we find,

$$
\Delta k_{j}\left(\rho+\rho^{h}\right)=\frac{\phi_{\uparrow}}{2 \pi L}+\int K_{1}(\sin k-\lambda) \Delta \lambda \sigma(\lambda) d \lambda
$$




$$
\begin{aligned}
& +\frac{1}{L} K_{1}(\sin k-\Lambda) \Delta \Lambda, \\
\Delta \lambda \sigma(\lambda)= & \frac{\phi_{\downarrow}-\phi_{\uparrow}}{2 \pi L}+\int K_{1}(\lambda-\sin k) \cos k \Delta k d k \\
& -\int K_{2}\left(\lambda-\lambda^{\prime}\right) \Delta \lambda^{\prime} \sigma\left(\lambda^{\prime}\right) d \lambda^{\prime} . \\
\Delta \Lambda \sigma^{\prime}(\Lambda)= & -\frac{\phi_{\downarrow}+\phi_{\uparrow}}{2 \pi L} \\
& -\int K_{1}(\Lambda-\sin k) \cos k \Delta k \rho(k) d k \\
& -\int K_{2}\left(\Lambda-\Lambda^{\prime}\right) \sigma^{\prime}\left(\Lambda^{\prime}\right) d \Lambda^{\prime},
\end{aligned}
$$

where $\sigma^{\prime}\left(\Lambda^{\prime}\right)=\delta\left(\Lambda^{\prime}-\Lambda\right) / L$. Integrations involving the variables $\lambda, k$, and $\Lambda$ lead to,

$$
\Delta \Lambda=\frac{1}{2}\left[\cos k_{1}^{h} \Delta k_{1}^{h}+\cos k_{2}^{h} \Delta k_{2}^{h}\right] .
$$

This is consistent with $\rho^{h}=\left[\delta\left(k-k_{1}^{h}\right)+\delta\left(k-k_{2}^{h}\right)\right] / L$ and $\Lambda=\left(\sin k_{1}^{h}+\sin k_{2}^{h}\right) / 2$.

The energy of the present elementary excitation is,

$$
E=-2 t L \int \cos k \rho(k) d k+4 t \mathcal{R} \sqrt{1-(\Lambda-i u)^{2}} .
$$

Thus, the corresponding energy deviation in the presence of the external flux can be expressed as,

$$
\Delta E(\phi)=2 t L \int \sin k \Delta k \rho(k) d k+4 t \mathcal{R} \frac{(\Lambda-i u) \Delta \Lambda}{\sqrt{1-(\Lambda-i u)^{2}}} .
$$

Note that the momentum of the two charge-distribution holes is determined by the value of the rapidity $\Lambda$. The important point is that their contributions to the charge-current cancel in the thermodynamical limit.

Numerical solution of the BA equations with the quantum-number occupancy configurations given in Eq. (49) leads to the energy spectra plotted in Fig. 8. From use of Eq. (5) we could confirm that both the spin and charge carried by these elementary excitations vanish in the thermodynamical limit [27].

\subsection{Half-filling with non-zero magnetization}

While the energy spectra plotted in figures 1-8 correspond to the general energy spectrum given in Eq. (15), in the figures 9-14 presented below the energy spectra of the elementary excitations refer to the general spectrum provided in Eq. (17).

Here we consider that the initial half-filling ground state corresponds to a finite spin density. That is achieved by the presence of a magnetic field. Thus, for such a ground state and corresponding elementary excitations one has that $M<N / 2$ and the integration limit of the spin variable $\lambda$ changes from $\infty$ to a finite cut-off. For odd values of $M$, the distribution of the corresponding spin quantum numbers is still of the form 

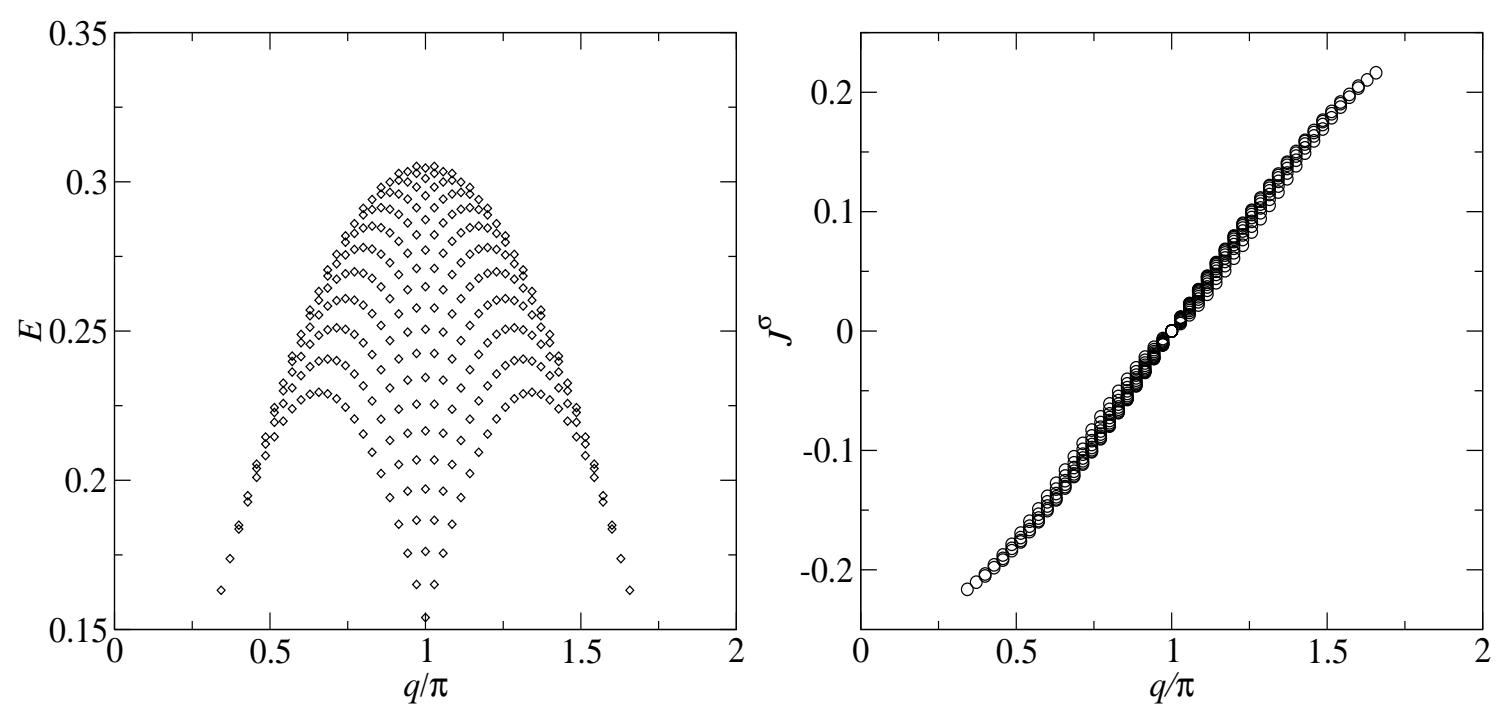

Figure 9. The half-filling energy spectrum (left) and spin-current spectrum (right)
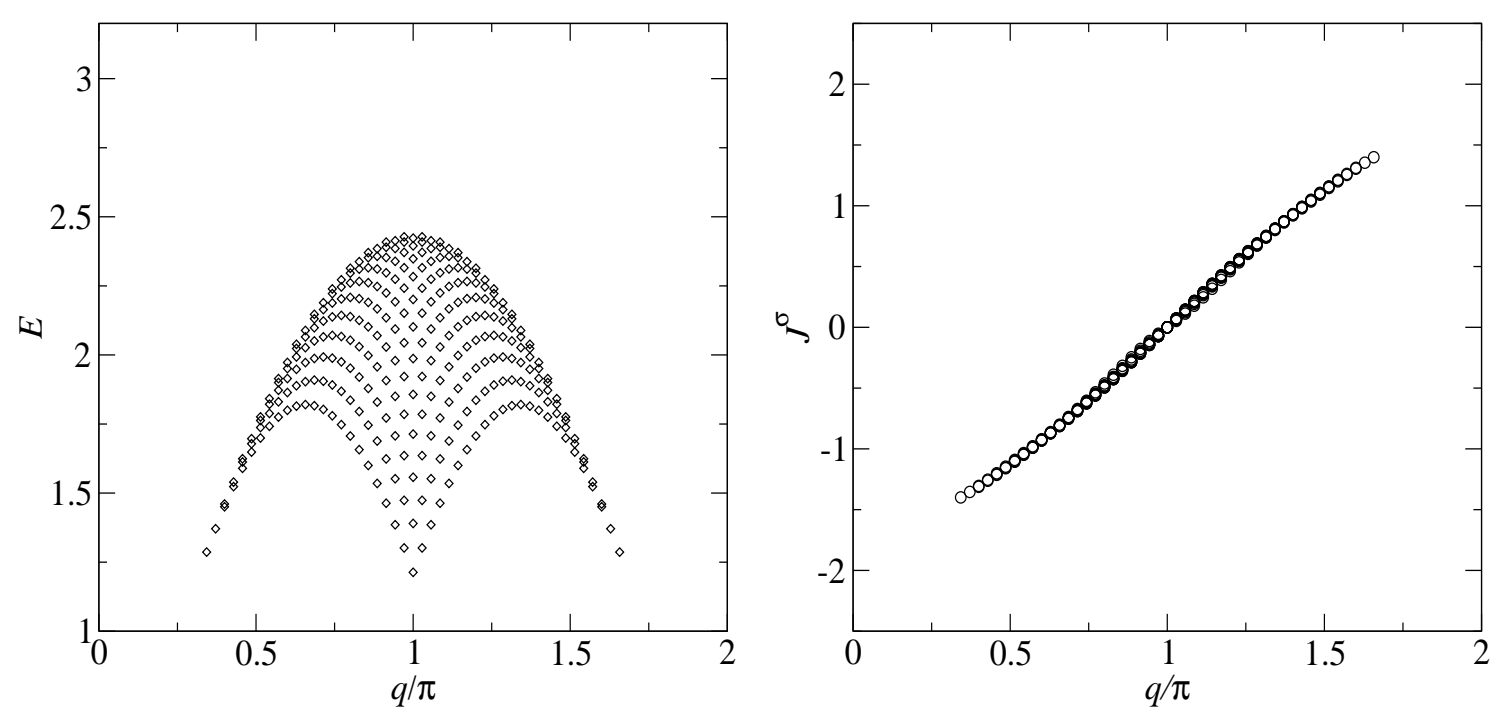

Figure 10. The half-filling energy spectrum (left) and spin-current spectrum (right) of the spin excitation with a string of length two for $u=1, N=L=75$, and $M=25$.

given in Eq. (16). The charge excitations do not change much due to the spin-charge separation. For the spin sector, however, the excitation spectrum presents qualitative changes.

There are still excitations which increase the value of $S$ by one. However, such excitations require a minimum finite energy and thus the corresponding excitation energy spectrum has a gap. The spin distribution of these elementary excitations displays two holes. Moreover, such elementary excitations carry in general a finite spin current. Additionally, since the integration limit of $\lambda$ is now finite, the external flux can 
shift the whole integration region. That process also contributes to the spin current. The corresponding current feature is expected to be a linear function of the momentum $q$. The same kind of spin-current feature arises now from elementary excitations involving one spin string of length two. For the case of the zero-magnetization ground state such excitation is of spin singlet character. However, in contrast to the zero-spin case now the two spin-distribution holes contribute to the spin current. Consideration of elementary excitations whose deviations from the quantum number configuration occupancies is the same as that of equations (33) for the spin singlet excitation, leads in the case of an initial ground state with finite spin density to the energy and spin-current spectra plotted in Figs. 9 and 10 for $u=10$ and $u=1$, respectively. Note that now such excitations lead indeed to a finite value for the spin current.

\subsection{Away from half filling}

Finally, let us consider that the initial ground state is metallic and thus refers to an electronic density away from half-filling. The ground-state spin density is considered to be zero. In this case, besides the ground state, there also exist real rapidity solutions for some of the elementary excitations. For simplicity, we still consider the case of $N / 2$ odd but now with $N<L$. The simplest elementary excitation away from half-filling corresponds to removing one $I_{j}$ from the ground-state occupied charge-distribution sector and adding a new one outside such a sector. Such an excitation has a "particlehole" character. It is characterized by the following BA numbers,

$$
\begin{aligned}
\left\{I_{j}\right\}= & \left\{-\frac{N-1}{2}, \ldots,-\frac{N-1}{2}+n-1,\right. \\
& \left.-\frac{N-1}{2}+n+1, \ldots, \frac{N-1}{2}, I_{n}\right\} .
\end{aligned}
$$

where $\left|I_{n}\right|>(N-1) / 2$. We consider excitations such that the $\lambda$ spin distribution remains unchanged.

In figures 11 and 12 we plot the energy and charge-current spectra of such elementary excitations for $u=10$ and $u=1$, respectively. Note that for strong coupling $(u=10)$ the charge current may have negative values whereas for weak coupling $(u=1)$ it has always positive values.

In the strong coupling limit the charge BA equation simplifies to,

$$
k_{j} L=2 \pi I_{j}+\phi_{\uparrow} .
$$

It follows that the energy deviation can be written as,

$$
\Delta E(\phi)=2 t \sum_{j} \sin k_{j} \Delta k_{j}
$$

Furthermore, the current reads,

$$
J^{\rho}=2 t\left[\sin \left(2 k_{F}+q\right)-\sin \left(2 k_{F}\right)\right],
$$

where $2 k_{F}=\pi N / L$ denotes the "Fermi momentum" and $q$ the momentum. Note that Eq. (58) provides the charge current for an excitation where a charge is removed from the 

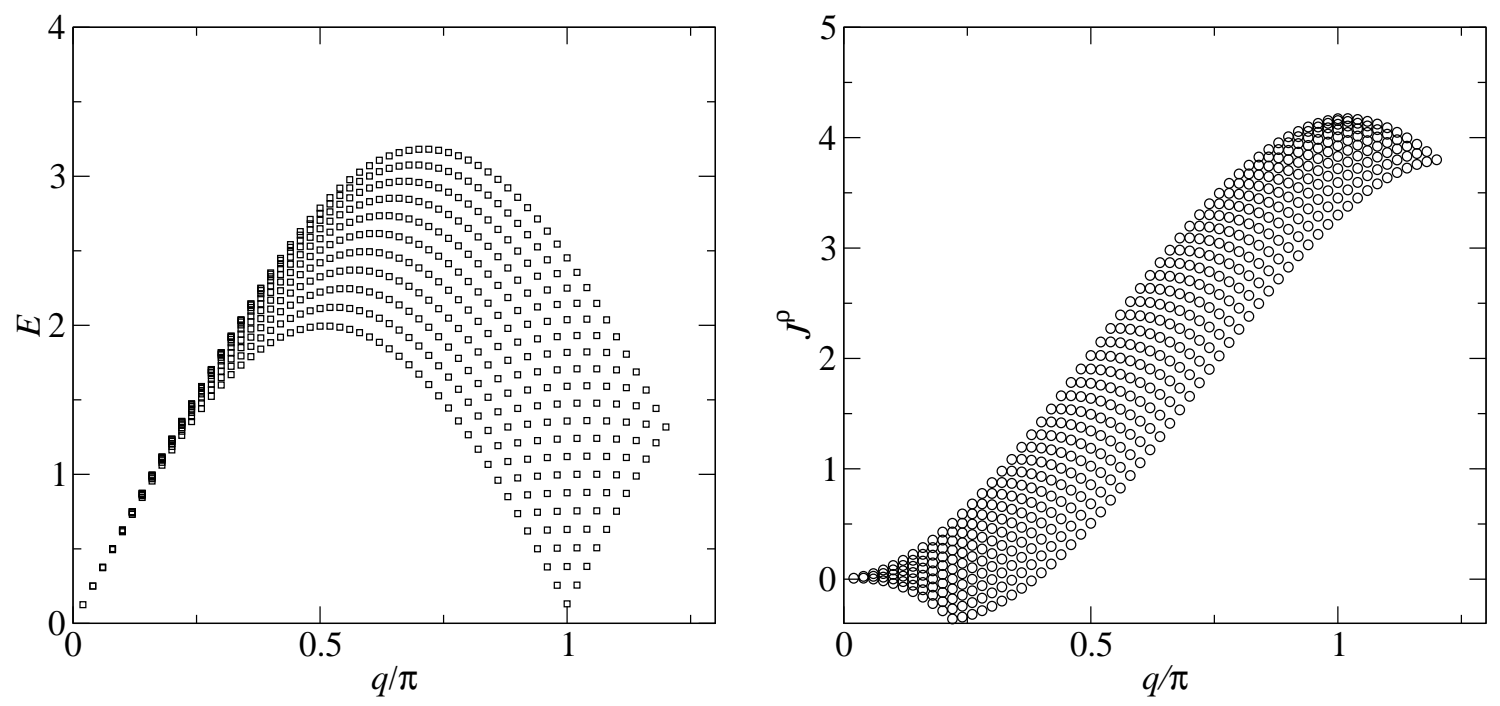

Figure 11. The quarter-filling energy spectrum (left) and charge current spectrum (right) of a charge "particle-hole" excitation for $u=10, L=100, N=50$, and $M=25$.
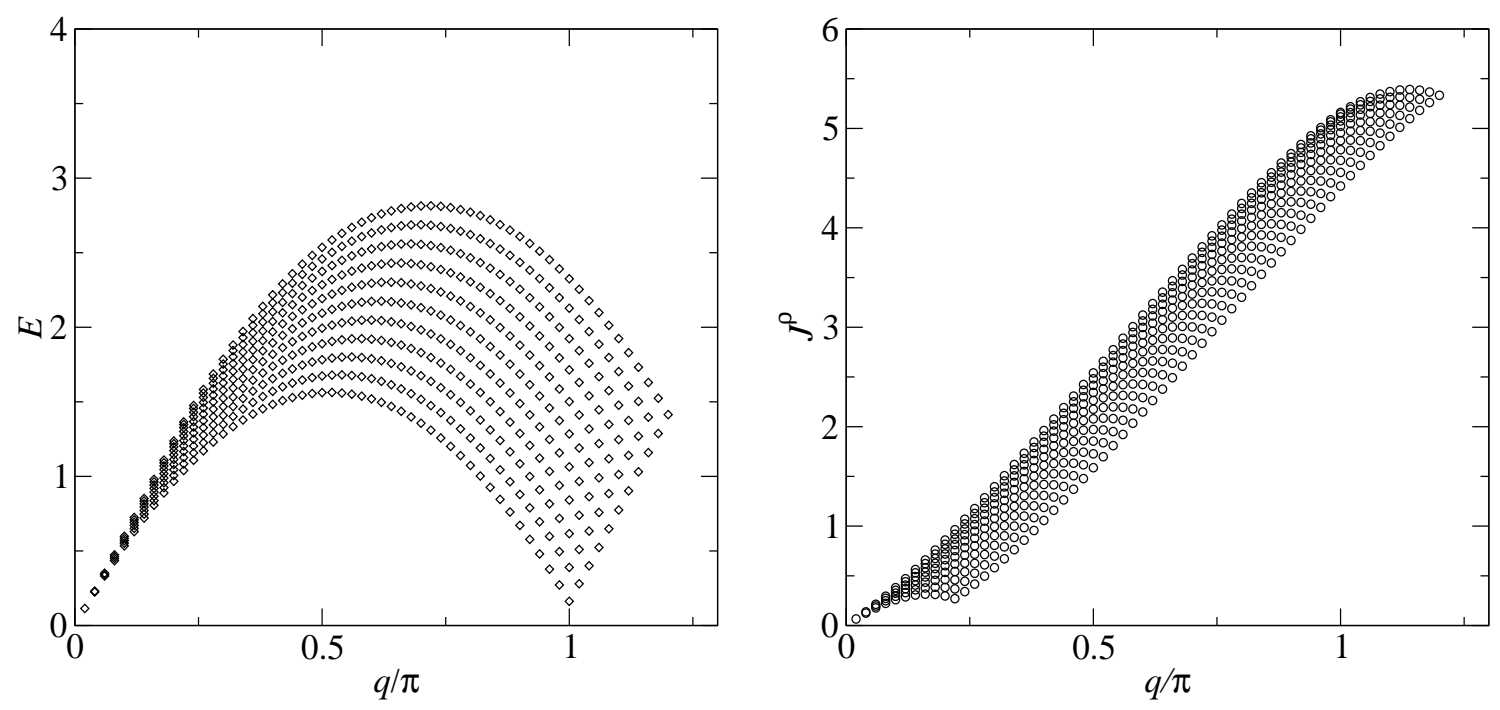

Figure 12. The quarter-filling energy spectrum (left) and charge current spectrum (right) of a charge "particle-hole" excitation for $u=1, L=100, N=50$, and $M=25$.

"Fermi point" and created outside the ground-state "Fermi sea". As long as $2 k_{F}>\pi / 2$, the current in the vicinity of $2 k_{F}$ is negative. On the other hand, the effective charge carried by this excitation is negative.

The contribution to the charge current of the charge-distribution hole excitation is straightforward to obtain and reads,

$$
J_{h}^{\rho}=2 t\left[\sin 2 k_{F}-\sin k_{h}\right], \quad k_{h} \in\left[-2 k_{F}, 2 k_{F}\right],
$$


where $k_{h}$ is the momentum of the charge-distribution hole. At $2 k_{F}=\pi / 2$ it leads to a feature in the charge current spectrum defined by the function $[1-\cos q / 2]$. Combining the "particle" and "hole" contributions one finds,

$$
J_{p}^{\rho}=2 t\left[\sin \left(2 k_{F}+k\right)-\sin k_{h}\right] .
$$

The group velocities of the charge "particle" and "hole" are such that,

$$
v_{p}^{\rho}=-2 t \sin k_{p}, \quad v_{h}^{\rho}=2 t \sin k_{h} .
$$

Hence, the effective charge carried by the "particle" and the "hole" are $e(=-1)$ and $-e(=1)$, respectively. We recall that all this analysis applies to the strong coupling limit only.

In turn, in the weak coupling limit the interaction between the charge holes is so weak in the "Fermi sea" that the charge current is almost a linear function of the momentum. For the "particle" at quarter filling, however, since the distribution function $\rho(k)$ becomes a very narrow peak and the "Fermi surface" is compressed, the charge current changes from negative to positive.

We have shown above that both the spin and charge currents carried by the charge $\eta$-spin-singlet excitations containing one charge string of length one vanish. However, away from half-filling the corresponding charge excitations containing one charge string of length one have different properties. The quantum number occupancy configuration of such elementary excitations is still described by Eq. (49). We have solved the corresponding BA equations. The found energy spectrum and charge-current spectrum are plotted in Figs. 13 and 14 for $u=10$ and $u=1$, respectively. In contrast to the corresponding excitations relative to the half-filling ground state, the present excitations carry charge but no spin.

\section{Concluding remarks}

In this paper we have studied the spin and charge currents carried by the elementary excitations of the 1D Hubbard model. Most of our results refer to half-filling. Both the charge $\eta$-spin-singlet and spin-singlet elementary excitations considered in our study carry no charge and no spin. Moreover, the spin-triplet excitations considered in this paper carry spin but no charge, while the charge $\eta$-spin-triplet elementary excitations carry charge but no spin.

Our results reveal that the present quantum liquid is not an ideal insulator for halffilling, in apparent contradiction to the general predictions of Ref. [4]. Indeed, ideal insulating behavior requires that all half-filling states carry no charge current. However, we note that out of the three types of charge $\eta$-spin-triplet elementary excitations of $\eta$-spin projections $0, \pm 1$, the states with projection 0 are indeed half-filling states. Our results show that such charge $\eta$-spin-triplet states carry finite charge current both in the strong and weak coupling limits. According to the results of Ref. [4], this implies a finite value for the charge stiffness $D(T)$ at finite temperatures $T>0$. This result agrees qualitatively with the studies of Ref. [8], which lead to a finite value for $D(T)$ at 

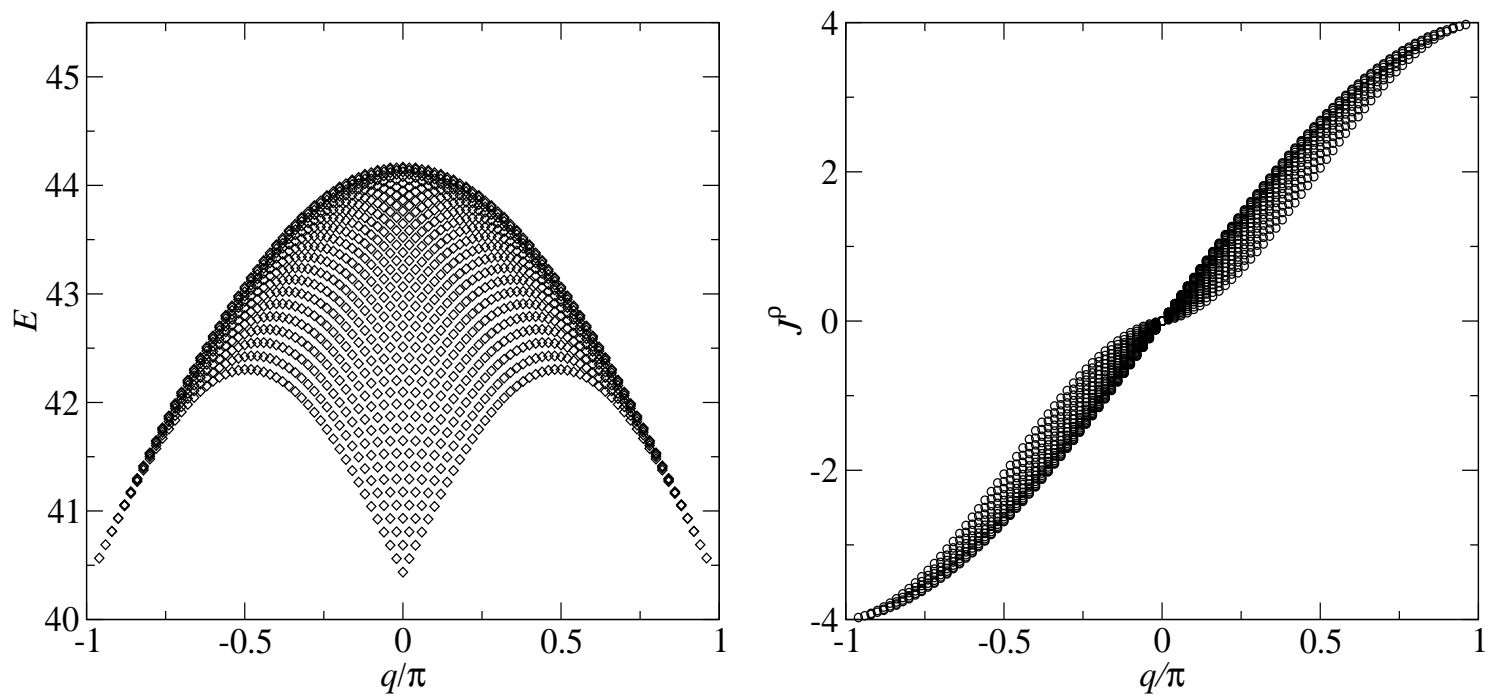

Figure 13. The quarter-filling energy spectrum (left) and charge-current spectrum (right) of a charge excitation containing one charge string of length one at for $u=10$,
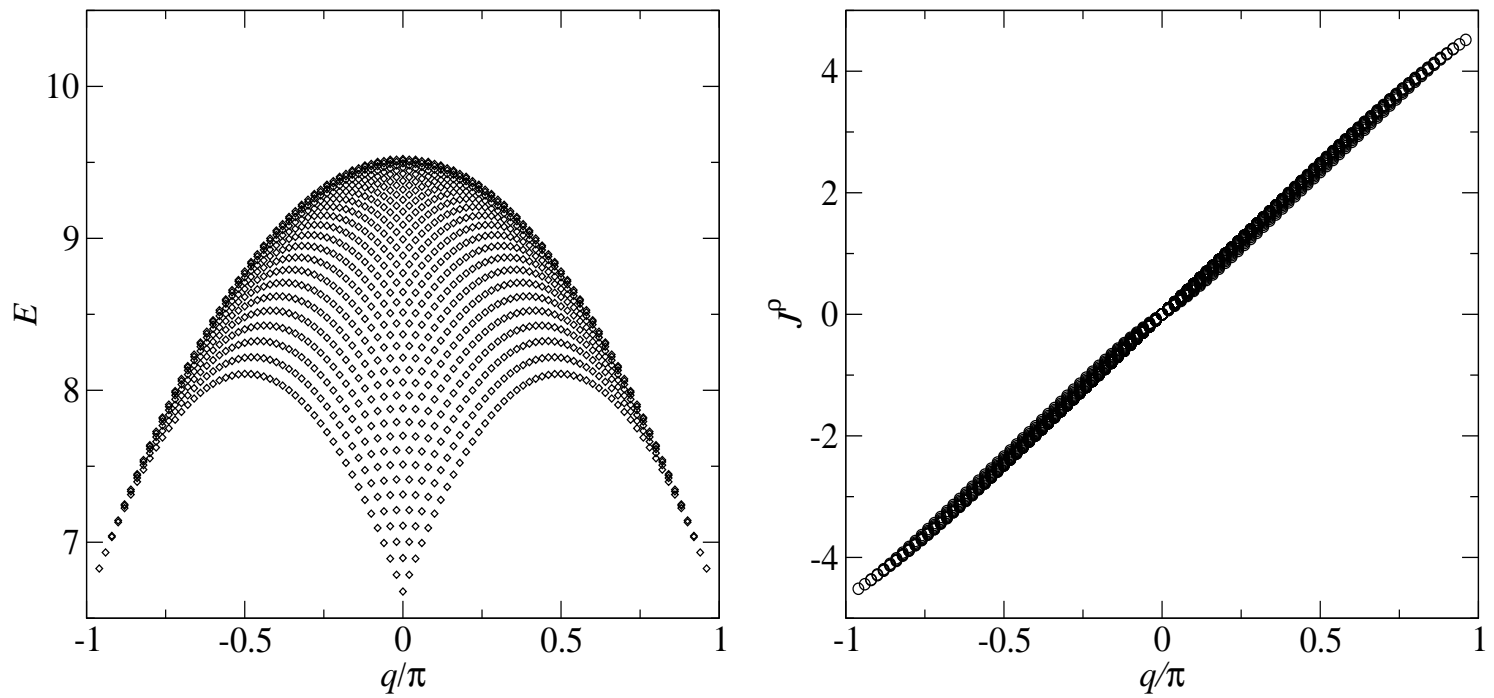

Figure 14. The quarter-filling energy spectrum (left) and charge-current spectrum (right) of a charge excitation containing one charge string of length one at for $u=1$, $L=100, N=50$, and $M=25$.

half filling and $T>0$. However, such studies did not take into account the $\eta>0$ and $\eta_{z}=0$ states which carry the charge current at half filling and lead to $D(T)>0$ for $T>0$. Moreover, both the studies of the present paper and the related studies of Ref. [27] provide strong evidence that the $\eta=0$ and $\eta_{z}=0$ states considered in Ref. [8] do not carry charge current and do not contribute to $D(T)$.

The model studied here is integrable and according to the general arguments of Ref. [4] should display ideal insulating behavior at half filling. That issue is clarified elsewhere 
by study of the microscopic mechanism which is behind the half-filling properties concerning transport of charge [27].

Finally, our results reveal the occurrence of charge-spin separation at finite energies, since some of the elementary excitations studied here have an energy gap relative to the initial ground state. Such a finite-energy charge-spin separation deserves further studies. We note that the photoemission studies of Ref. [2] have detected a spin-charge separation in quasi-1D compounds for the whole energy bandwidth.

\section{Acknowledgments}

S. J. G. thanks the support of the University of Évora where this research was initiated and acknowledges the financial support of Earmarked Grant for Research from the Research Grants Council of HKSAR, China (Project No CUHK 400906). J. M. P. C. and N. M. R. P. thank the support of the ESF Science Program INSTANS and grants POCTI/FIS/58133/2004 and PTDC/FIS/64926/2006.

\section{References}

[1] Ishii H et al. 2003 Nature 426540 Auslaender O M et al. 2005 Science $\mathbf{3 0 8} 88$ Lorenz T et al. 2002 Nature $\mathbf{4 1 8} 614$

[2] Claessen R, Sing M, Schwingenschlögl U, Blaha P, Dressel M, and Jacobsen C S 2002 Phys. Rev. Lett. 88096402

[3] Eckern U and Schwab P 2002 J. Low Temp. Phys. 1261291

[4] Castella H, Zotos X, and Prelovšek P 1995 Phys. Rev. Lett. 74972 Zotos X and Prelovšek P 1996 Phys. Rev. B 53983

[5] Hubbard J 1963 Proc. R. Soc. (London) A 276238

[6] Lieb E H and Wu F Y 1968 Phys. Rev. Lett. 201445

[7] Takahashi M 1972 Prog. Theor. Phys. 4769

[8] Fujimoto S and Kawakami N 1998 J. Phys. A: Math. Gen. 31465

[9] Peres N M R, Carmelo J M P , Campbell K K and Sandvik A W 1997 Zeitschrift für Physik B 103217 Martins M J and Fye R M 1991 J. Stat. Phys. 64271

[10] Li Y Q and Gruber C 1998 Phys. Rev. Lett. 801034 Martins M J and Ramos P B 1998 Nucl. Phys. B 522413

[11] Bethe H A 1931 Z. Physik 71205

[12] Baeriswyl D, Carmelo J and Maki K 1987 Synthetic Metals 21271.

[13] Sing M it al. 2003 Phys. Rev. B 68125111

[14] Yang C N 1967 Phys. Rev. Lett. 191312

[15] Gaudin M 1967 Phys. Lett. A 2455

[16] Frahm H and Korepin V E 1990 Phys. Rev. B 4210553 Frahm H and Korepin V E 1991 Phys. Rev. B 435653

[17] Schulz H J 1990 Phys. Rev. Lett. 642831

[18] Carmelo JMP, Castro Neto AH and Campbell DK 1994 Physical Review Letters 73926.

[19] Carmelo J M P and Horsch P 1992 Phys. Rev. Lett. 68871 Carmelo J M P, Horsch P, and Ovchinnikov A A 1992 Phys. Rev. B 4614728

[20] Stafford C A and Mills A J 1993 Phys. Rev. B 481409 
[21] Heilmann O J and Lieb E H 1971 Ann. N. Y. Acad. Sci. 172583

Lieb E H 1989 Phys. Rev. Lett. 621201

[22] Yang C N 1989 Phys. Rev. Lett. 632144

[23] Yang C N and Zhang S C 1990 Mod. Phys. Lett. B 440

[24] Deguchi T, Essler F H L, Göhmann F, Klümper A, Korepin V E, and Kusakabe K 2000 Phys. Rep. 331197

[25] Essler F H L, Korepin V E, and Schoutens K 1992 Nucl. Phys. B 384431

[26] Shastry B S and Sutherland B 1990 Phys. Rev. Lett. 65243

[27] Carmelo J M P, Gu S J, and Peres N M R 2007 Europhys. Lett. 7817005

[28] Peres N M R, Dias R G, Sacramento P D, and Carmelo J M P 2000 Phys. Rev. B 615169

[29] Peres N M R, Sacramento P D and Carmelo J M P 2001 J. Phys: Condens. Matter 135135

[30] Takahashi M 1999 Thermodynamics of One-Dimensional Solvable Models (Cambridge: Cambridge University Press)

[31] Zhang Q L and Gu S J 2007 Chin. Phys. Lett. 241354 